\title{
EFFICACY OF THE SMR PROTOCOL IN WOMEN WITH FIBROMYALGIA FOR THE IMPROVEMENT OF CHRONIC PAIN, SLEEP AND QUALITY OF LIFE
}

\author{
Carlos Barbosa-Torres and Sixto Cubo-Delgado \\ University of Extremadura (Spain)
}

\begin{abstract}
The objective of the study was to analyze how the brain shaping provided by the sensorimotor rhythm protocol (SMR), applied on somatosensory areas, affects pain, sleep and the quality of life in women with fibromyalgia. Thirty-seven women with fibromyalgia who received an SMR protocol in 20 sessions participated and were evaluated before and after treatment. The data showed an increase in the amplitude of the SMR $(p=.026)$ and a decrease in the amplitude of the theta band $(p=.011)$ in the somatosensory cortex after the application of therapy, which caused an increase in the SMR/theta ratio $(p=.048)$. In addition, the scores on the Chronic Pain Scale $(p=.002)$, the Pittsburgh Sleep Quality Index $(p=.001)$, and the SF-36 Health Survey $(p=.000)$ improved significantly. The SMR protocol applied to the somatosensory cortex favors the shaping of SMRs, which has an impact on stimulating the inhibition of the central nervous system of patients with fibromyalgia, improving symptoms such as pain, sleep and quality of life.

KEY WORDS: fibromyalgia, sensorimotor rhythms, theta waves, somatosensory cortex, chronic pain, sleep, quality of life.
\end{abstract}

\section{Resumen}

El objetivo del estudio fue analizar cómo el moldeamiento cerebral proporcionado por el protocolo de ritmos sensoriomotores (SMR), aplicado sobre áreas somatosensoriales, afecta al dolor, al sueño y a la calidad de vida de mujeres con fibromialgia. Participaron 37 mujeres con fibromialgia a quienes se les aplicó un protocolo de SMR durante 20 sesiones y fueron evaluadas antes y después del tratamiento. Los datos mostraron un aumento de la amplitud de los SMR ( $p=$ $0,026)$ y una disminución de la amplitud de la banda zeta $(p=0,011)$ en la corteza somatosensorial tras la aplicación de la terapia, lo que provocó un aumento de la ratio SMR/zeta $(p=0,048)$. Además, mejoraron significativamente las puntuaciones en la "Escala de dolor crónico" $(p=0,002)$, el "Índice de calidad del sueño de Pittsburgh" $(p=0,001)$ y la "Encuesta de salud" (SF-36) $(p=0,000)$. El protocolo SMR aplicado en la corteza somatosensorial favorece el moldeamiento de los SMR, lo que repercute en la inhibición estimular del sistema nervioso central de los

The authors are grateful for the collaboration of the professionals of the Pain Unit of the Perpetual Socorro Hospital in Badajoz.

Correspondence: Carlos Barbosa Torres, Faculty of Education and Psychology, University of Extremadura, Av. Elvas, s/n, 06006 Badajoz (Spain). E-mail: carlosbarbosa@unex.es 
pacientes con fibromialgia mejorando síntomas como el dolor, el sueño y la calidad de vida.

Palabras clave: fibromialgia, ritmos sensoriomotores, ondas zeta, corteza somatosensorial, dolor crónico, sueño, calidad de vida.

\section{Introduction}

Fibromyalgia syndrome is a pathology of unknown origin that mainly affects women. The clinical manifestation of fibromyalgia is characterized by musculoskeletal pain of non-articular origin, sleep problems, chronic fatigue, hyperalgesia and problems in cognition, and other symptoms mediated by the central nervous system have a considerable impact on the quality of life (Aparicio, et al., 2013; Cabo-Meseguer, 2017; Wolfe et al., 2016). Fibromyalgia syndrome presents a multidisciplinary treatment that ranges from the use of drugs of diverse natures, such as serotonin-norepinephrine reuptake inhibitors (SNRIs) (Bidari et al., 2019) and tricyclic antidepressants (Thorpe, Shum, Moore, Wiffen, \& Gilron, 2018), to cognitive therapies (Bernardy, Fuber, Kollner, \& Hauser 2010), physical training (Bidonde et al., 2017) and neurofeedback (Kayıran, Dursun, Dursun, Ermutlu, \& Karamürsel, 2010; Sánchez, Nakakaneku, Miró \& Martínez, 2021).

Central sensitization syndrome (CSS) is the most widespread hypothesis to explain this disease. CSS consists of an increase in sensitization and a decrease in stimulus thresholds as well as the maintenance of both painful and non-painful symptoms over time (Yunus, 2012). Other studies support that patients with fibromyalgia present problems in sensory processing and that the deactivation of inhibitory mechanisms can be considered relevant in the functioning of the pathology (Napadow, 2012).

In patients with fibromyalgia, the presence of pain is shown by the increase in theta waves $(4-7 \mathrm{~Hz})$ and the decrease in sensorimotor rhythms $(12-15 \mathrm{~Hz})$. The training carried out through operant conditioning, with a sensorimotor rhythm protocol (SMR), could facilitate the reorganization of the intrinsic pathways found in the sensory amplification and neurosensitization systems of pain as well as other types of stimuli. The objective of this training is to interrupt the non-adaptive processes, to regulate the flow of information and to reduce symptoms such as chronic pain (Egner \& Gruzelier, 2001; Caro \& Winter, 2011). When sensorimotor rhythms occur, the discharge pattern of the ventrobasal nuclei changes from rapid and nonrhythmic to rhythmic and systemic shocks. This event is associated with the suppression of the passage of somatosensory information and the reduction in muscle tone (Howe, 1972). However, this pattern is diminished when patients suffer disorders such as anxiety, stress, migraines, chronic pain, and attention or sleep problems (Kober et al., 2015).

The SMR protocol applied with a neurofeedback technique makes it possible to work at the cortical level and reprogram some of the information pathways involved in this process for the relief of pain and sleep, among other symptoms (Jensen, Hakimian, Sherlin, \& Fregni, 2008; Lovato, Miller, Gordon, Grunstein, \& Lack, 2018). Generalized chronic pain is biographically disruptive and significantly 
affects the quality of life of patients who suffer from it, producing serious repercussions on a physical, social and occupational level, especially in patients with fibromyalgia (Costa et al., 2017).

Another influential variable in the pain process is lack of sleep, which can reduce the threshold of this stimulus, causing hyperalgesia in patients who have already presented this symptom. Sleep has traditionally been used as a significant predictor not only of pain but also of chronic fatigue and maladaptive social functioning in fibromyalgia (Prados \& Miró, 2012). The objective of this study is to analyze how the brain autoregulation provided by the sensorimotor rhythms protocol, which is designed to increase sensorimotor rhythms and to decrease theta waves on somatosensory areas, affects patients with fibromyalgia. With this training, it is intended that patients adapt to therapy and, therefore, a greater amplitude of the SMR/theta ratio, which would improve perceived pain, health-related quality of life and sleep after therapy.

\section{Method}

\section{Participants}

The study was carried out with a total sample of 37 women, with a mean age of 54.92 years $(S D=7.9$ ), selected thanks to the help of the Pain Unit of the Perpetuo Socorro Hospital in Badajoz. The inclusion criteria were characterized by presenting the diagnosis of fibromyalgia syndrome according to the criteria established by the American College of Rheumatology (Wolfe, 2016), having an age between 18 and 65 years and not having any diagnosis or chronic disease such as cancer, epilepsy or alcoholism that could limit the effectiveness of this type of therapy. Informed consent about the study objective and information on the neurofeedback technique were provided to all the patients. Table 1 shows all the sociodemographic variables studied.

\section{Instruments}

a) Chronic Pain Scale (CPS; Von Korff, Ormel, Keefe, \& Dworkin, 1992) Spanish version of Ferrer-Peña et al. (2016). The CPS was used to assess the magnitude of chronic pain. It consists of 7 items in 11-point Likert format, ranging from "no pain" to "the worst pain imaginable" with a range from 0 to 70 points, where the highest scores indicate greater pain perception. Scores below and above 35 have been used to indicate better or worse states of perceived pain, respectively. This scale has a high internal consistency of 0.87 , similar to the versions in other languages, and the intraclass correlation coefficient is 0.81 for the Spanish version (Ferrer-Peña, et al., 2016). In our sample of women diagnosed with fibromyalgia, a Cronbach's $\alpha$ of 0.75 was obtained. 
Table 1

Characteristics of the patients $(N=37)$

\begin{tabular}{|c|l|}
\hline \multicolumn{1}{|c|}{ Variables } & \multicolumn{1}{|c|}{$n(\%)$} \\
\hline Pain symmetry & $24(64.8)$ \\
\hline Yes & $13(35.2)$ \\
\hline No & \\
\hline Family members with fibromyalgia & $1(2.7)$ \\
\hline Mothers/fathers & $6(16.2)$ \\
\hline Brothers/sisters & $30(81.1)$ \\
\hline None & \\
\hline Sick leave & $12(32.4)$ \\
\hline Yes, at this moment & $7(18.9)$ \\
\hline No, at this moment & $18(48.7)$ \\
\hline Never & \\
\hline Level of studies & $14(37.8)$ \\
\hline Primary studies & $15(40.6)$ \\
\hline Mid-level studies & $8(21.6)$ \\
\hline Higher education & \\
\hline Civil status & $28(75.6)$ \\
\hline Married & $5(13.6)$ \\
\hline Divorced / separated & $4(10.8)$ \\
\hline Widows & \\
\hline Socioeconomic level & $23(62.1)$ \\
\hline Low & $13(35.2)$ \\
\hline Half & $1(2.7)$ \\
\hline High & \\
\hline
\end{tabular}

b) SF-36 Health Survey (SF-36; Ware \& Sherbourne, 1992), Spanish version of Alonso et al. (1998). The SF-36 was used to measure health-related quality of life. This questionnaire measures health in 8 dimensions (physical functioning, physical role, emotional role, social functioning, pain, general health, vitality and mental health), and a final dimension called "health transition," with a range between 0 points, "the worst possible health", and 100 points, "the best possible health." The cut-off point of 50 has been used to indicate "worse health." The questionnaire shows an internal consistency range of 0.78-0.96 for all dimensions (Alonso et al., 1998). In our sample, an internal consistency range (Cronbach's $\alpha$ ) was obtained between 0.66 and 0.91 for all dimensions of the questionnaire, with the dimension "social function" being the lowest and with the dimension "physical role" being the highest.

c) Pittsburgh Sleep Quality Index (PSQI; Buysse, Reynolds, Monk, Berman, \& Kupfer, 1989) Spanish version of Royuela \& Macías (1997). The PSQI was used to assess sleep quality, specifically seven dimensions (subjective sleep quality, sleep latency, sleep duration, sleep efficiency, sleep disorders, use of sleep medications, and daytime dysfunction). The 19 items of the PSQI are answered on a 4-point Likert scale (from $0=$ there are no problems in this regard to $3=$ serious problems), and scores above 5 indicate significant sleep interruptions. 
This test showed high internal consistency using Cronbach's $\alpha$ of 0.81 as well as high sensitivity (88.63\%) and specificity of $74.19 \%$ in the Spanish population (Royuela \& Macías, 1997). In the present study, an internal consistency range (Cronbach's $\alpha$ ) of 0.64-0.78 was obtained for our sample, with the dimension "drug use" being the lowest and with the dimension "sleep efficiency" being the highest.

d) FlexComp Infiniti Encoder EEG (Thought Technolog, 2021). The EEG was used to collect and analyze the electrophysiological variables. The main measurement variables were the amplitude of the SMR $(12-15 \mathrm{~Hz})$, the theta bands $(4-8 \mathrm{~Hz})$, and the ratio between both produced variables before and after the therapy sessions. The signal was analyzed at 256 samples per second with a monopolar C4 spoon electrode assembly according to the international 10-20 system, with the reference electrode placed on the left ear and the ground electrode placed on the right ear lobe, an impedance verification below $5 \mathrm{~K} \omega$ during therapy, and an EEG sensor supply voltage of $7.260 \mathrm{~V} \pm 1.969 \mathrm{~V}$ and a $3.6 \mathrm{~V}-6.5 \mathrm{~V}$ fiber optic supply voltage between the sensor junction and the device. The software used to capture and analyze the neurofeedback data was the BioGraph Infiniti, which has a validated protocol to analyze and feedback the SMRs.

\section{Procedure}

We conducted a study from the perspective of the quantitative paradigm with a single-group quasi-experimental design with a non-randomized pre-test and posttest.

The standardized SMR protocol used was applied 3 times a week until completing 20 sessions of 15 minutes each, in addition to approximately 5 minutes for the placement of the electrodes. The interval between the pre-test and the posttest in the group of patients was between 6 and 7 weeks. The protocol developed with the patients was as follows:

1) The training was carried out in an isolated room free from external stimuli.

2) The patients sat comfortably in front of a screen, where they were informed with clear and open instructions to maximize the results of the therapy.

3) The instructions consisted of explaining to the participants that they should concentrate on the puzzle that would appear piece by piece on the screen depending on their concentration.

4) On the screen, visual and auditory stimuli provided by the software appeared, that is, the pieces of the puzzle, an associated beep for each piece and the amplitude values of the waves transformed into visual representations. These stimuli were used as reinforcement or feedback stimuli for the patients.

5) Once the first puzzle was finished, another new puzzle appeared that they had to complete in the same way, continuing this dynamic until the end of the 15minute session.

All of the procedures carried out in this work complied with the safety regulations for the application of this type of therapy as well as all the ethical 
standards of the relevant institutional committee on human experimentation set out in the Declaration of Helsinki.

\section{Data analysis}

To perform the inferential analysis, we used the following process. The ShapiroWilk test applied to test the theoretical population was normal in contrast to the null hypothesis, and the runs test was performed to check that the null hypothesis of the theoretical distribution in the population was random. The Levene's test was not performed to contrast the null hypothesis related to the equality of the variances between the different variables analyzed because they were related samples. After verifying these assumptions, the nonparametric Wilcoxon signed-rank test was applied to analyze the pre-test and post-test of all variables, and Cohen's d was used to analyze the effect size.

\section{Results}

Regarding the inferential analysis of the measured physiological variable, the data showed an increase in the amplitude of sensorimotor rhythms $(p=.026)$ and a decrease in the amplitude of theta waves $(p=.011)$, both significant, in the somatosensory cortex. This brain wave shaping process was also observed in the $S M R / t h e t a$ ratio, which showed a significant increase in its amplitude after the application of neurofeedback therapy in the same region $(p=.048)$.

In relation to the other measures, the total score of the Chronic Pain Scale (CPS) decreased significantly $(p=0.002)$. There was a decrease in the number of patients who scored above the cut-off point in the pre-test $(97.3 \%)$ in relation to the posttest $(72.9 \%)$.

The total score of the SF-36 Health Survey also showed a significant increase $(p=.000)$ in the total score and in its dimensions, except in the dimension "social functioning" $(p=.152)$. There was a significant decrease in the pre-test below the cut-off point (91.8\%) compared with the post-test (64.9\%).

The total score of the Pittsburgh Sleep Quality Index (PSQI) decreased significantly $(p=.001)$ as well as all of its dimensions except "subjective quality of sleep," which, despite its decrease, did not show significant results $(p=.116)$. In this last questionnaire, the number of patients who exceeded the cut-off point of the pre-test $(35.1 \%)$ was reduced in comparison with the post-test $(10.8 \%)$.

The pre-test and post-test results of the questionnaires are shown in Table 2, and the mean scores and their standard deviations, the degree of significance, and the effect size (Cohen's d) are shown in Table 3. 
Table 2

Results in relation to the cut-off points in the pre-test and post-test analysis of the questionnaires

\begin{tabular}{|l|c|c|c|c|c|}
\hline \multirow{2}{*}{ Instrument } & Measured & \multicolumn{2}{|c|}{$\leq$ Cut point $^{*}$} & \multicolumn{2}{|c|}{$>$ Cut point * } \\
\cline { 3 - 6 } & moment & $n(\%)$ & $M(S D)$ & $n \mathrm{n}(\%)$ & $M(\mathrm{SD})$ \\
\hline \multirow{2}{*}{ CPS } & Pre-test & $1(2.7)$ & $9(7.56)$ & $36(97.3)$ & $53.20(12.43)$ \\
\cline { 2 - 6 } & Post-test & $10(27.1)$ & $24.10(9.46)$ & $27(72.9)$ & $53.07(10.78)$ \\
\hline \multirow{2}{*}{ SF-36 } & Pre-test & $34(91.8)$ & $31.06(12.78)$ & $3(8.2)$ & $69.4(14.77)$ \\
\cline { 2 - 6 } & Post-test & $24(64.9)$ & $38.06(10.41)$ & $13(35.1)$ & $58.07(11.59)$ \\
\hline \multirow{2}{*}{ PSQI } & Pre-test & $24(64.9)$ & $3.52(1.97)$ & $13(35.1)$ & $5.01(1.68)$ \\
\cline { 2 - 6 } & Post-test & $33(89.2)$ & $2.9(1.24)$ & $4(10.8)$ & $5.4(2.14)$ \\
\hline
\end{tabular}

Notes: CPS= Chronic Pain Scale; PSQI= Pittsburgh Sleep Quality Index; SF-36= SF-36 Health Survey. ${ }^{\star}$ Cut point: $\mathrm{PCS}=35 ; \mathrm{SF}-36 \mathrm{~V} 2=50 ; \mathrm{PSQl}=5$.

Table 3

Pre-test and post-test results of the questionnaires and physiological variables

\begin{tabular}{|l|c|c|c|c|c|}
\hline \multirow{2}{*}{ Variables } & \multicolumn{2}{|c|}{$M(\mathrm{SD})$} & Wilcoxon & \multirow{2}{*}{$\begin{array}{c}\text { Cohen's } \\
d\end{array}$} \\
\cline { 2 - 4 } & Pre-test & Post-test & $t$ & & .520 \\
\hline CPS & $52.05(12.16)$ & $45.41(14.33)$ & -3.957 & .002 & .787 \\
\hline SF-36 & $34.17(15.10)$ & $45.09(12.52)$ & -4.086 & .000 & .783 \\
\hline Physical functioning & $30.54(17.74)$ & $37.43(20.15)$ & -3.071 & .033 & .363 \\
\hline Physical role & $15.54(29.66)$ & $29.86(32.71)$ & -3.619 & .004 & .459 \\
\hline Emotional role & $51.34(40.51)$ & $70.25(39.13)$ & -2.551 & .011 & .475 \\
\hline Social functioning & $44.05(25.42)$ & $52.29(23.44)$ & -1.515 & .152 & .337 \\
\hline Pain & $24.71(17.85)$ & $39.28(19.15)$ & -3.619 & .000 & .841 \\
\hline General health & $23.71(13.51)$ & $32.29(13.25)$ & -3.101 & .005 & .641 \\
\hline Vitality & $27.16(18.31)$ & $41.08(17.76)$ & -4.020 & .000 & .772 \\
\hline Mental health & $52.54(12.89)$ & $58.00(12.76)$ & -2.314 & .042 & .426 \\
\hline Health transition & $28.37(26.45)$ & $45.27(25.57)$ & -2.818 & .003 & .650 \\
\hline PSQI & $4.04(.85)$ & $3.16(1.14)$ & -3.789 & .001 & .875 \\
\hline $\begin{array}{l}\text { Subjective quality of } \\
\text { sleep }\end{array}$ & $0.62(0.13)$ & $0.54(0.17)$ & -1.679 & .116 & .479 \\
\hline Sleep latency & $0.74(0.02)$ & $0.88(0.07)$ & -4.575 & .000 & .627 \\
\hline Sleep duration & $0.57(0.18)$ & $0.70(0.18)$ & -4.335 & .001 & .480 \\
\hline Sleep efficiency & $0.53(0.22)$ & $0.40(0.18)$ & -2.614 & .010 & .647 \\
\hline Sleep disorders & $0.63(0.19)$ & $0.46(0.21)$ & -3.754 & .002 & .849 \\
\hline Medication use & $0.93(0.17)$ & $0.80(0.10)$ & -3.802 & .001 & .932 \\
\hline Daytime dysfunction & $0.75(0.09)$ & $0.63(0.21)$ & -2.583 & .011 & .681 \\
\hline SMR & $7.082(3.93)$ & $7.988(3.29)$ & -2.874 & .026 & .252 \\
\hline Theta & $13.56(8.36)$ & $11.80(5.83)$ & -2.647 & .011 & .244 \\
\hline SMR/theta & 0.4827 & 0.5173 & -2.226 & .048 & .213 \\
\hline
\end{tabular}

Notes: CPS $=$ Chronic Pain Scale; PSQI= Pittsburgh Sleep Quality Index; SF-36= SF-36 Health Survey.; SMR/theta= sensorimotor rhythms/theta ratio. 


\section{Discussion}

The aim of the study was to examine the changes in SMR/theta potential in the somatosensory areas of patients with fibromyalgia applying an SMR protocol. The conclusion is that neurofeedback training under these conditions generated a significant reduction in chronic pain and an improvement in sleep and quality of life.

The increase in the SMR and the decrease in theta, which produces an increase in the amplitude of the SMR/theta ratio, favors the improvement of the inhibitory processes of the central nervous system as a result of the therapy, which generates a greater processing capacity, as other studies have shown (Sterman, 2000). This can also be inferred because the increase in SMR is associated with the increase in the evoked potential P300, which is an indicator of the activation of inhibitory processes (Egner \& Gruzelier, 2001). Therefore, it can be stated that the protocol SMR favors the general balance in brain functions in patients with fibromyalgia since it affects the entire spectrum to stimulate as explained by the CSS (Yunus, 2012).

These findings are not sufficient to establish that the SMR protocol can increase the inhibitory response of the central nervous system by itself. To reach these conclusions, it is necessary to record the metabolic activity during the synchronization and desynchronization of the bands through fMRI, to analyze the changes in the location of the waves, to accompany them with evoked potentials tests and to relate all these data with the main findings of our study, as recent research using these techniques has shown (Terrasa \& Barros, 2020). This study shows that patients who adapt well to SMR training can significantly reduce pain and can increase both SMR power modulation and the functional connectivity of motor and somatosensory areas during neurofeedback training.

These changes in brain activity, which seem to directly affect the perceived pain, are reflected in other variables, such as sleep and quality of life. Chronic pain and sleep quality decreased by $24.4 \%$ and $24.3 \%$, respectively, and the quality of life increased by $26.9 \%$. In addition, the number of patients who improved their scores in relation to the cut increased. Regarding this last variable, the scores of all the dimensions of quality of life showed significant improvement, except for "social functioning," which refers to limitations in social activities due to physical or emotional problems. This can probably be explained by the negativistic nature attributed to patients with fibromyalgia (Seto et al., 2019) and/or to the low level of internal consistency of this dimension in most of the adaptations being the lowest range (0.69-0.79) in all validations (Vilagut et al., 2005). If we compare the scores obtained in the SF-36 of this study and the study by Terrasa and Barros (2020), the scores found in the present study show fewer differences with the group of good responders to SMRs than with the group of poor responders to SMR training. Furthermore, in relation to the effect size, the statistic shows a lower effect than the results provided in our study, which could be explained by the greater number of sessions in our study.

Sleep disorders in fibromyalgia have been defined as difficulty falling asleep and staying asleep, reductions in sleep time, multiple interruptions at night and a lack of restful sleep (Moldofsky, 2002). In our study, all the dimensions of the questionnaire related to sleep showed significant improvement, excluding the 
dimension of "subjective quality of sleep", which could be related to comorbidity with other disorders or with certain personality characteristics (Seto et al., 2019; Clauw, 2014). Sleep quality is used as a predictor of pain, fatigue and maladaptive social functioning (Miró et al., 2011; Prados \& Miró, 2012). Therefore, a relationship could be established between the "subjective quality of sleep" dimension of the sleep variable and non-significant results of the "social functioning " dimension of the quality of life variable in future studies.

This study presents results consistent with the few investigations conducted with a sensorimotor rhythm protocol in patients with fibromyalgia where electrophysiological measures; physical measures, such as pain, fatigue and sensitivity; and psychological measures, such as attention, anxiety and depression were taken, which improved significantly after the training (Caro \& Winter, 2011; Kayıran, Dursun, Dursun, Ermutlu and Karamürsel, 2007; Kayıran et al., 2010; Terrasa \& Barros, 2020). The main common elements of these studies are the evaluation of the grading of chronic pain and the use of standardized SMR protocols. However, there are notable differences that mark the nature of the research. For example, in our study, the sample size was much larger; the activity provided by the software was different, although it consumed the same type of attentional resources; and the novel measurements were provided regarding the sleep variable.

According to our results, neurofeedback did not eradicate symptoms but significantly improved them by increasing the SMR/theta ratio. This can be explained by patient learning and their ability to adapt to the demands of neurofeedback (1) due to the reinforcing power of the task and (2) due to the reduction in external nociceptive stimulation that is generated, produced during training, and that can benefit changes in the central nervous system (Gwilym et al. 2011).

It is highly likely that the questionnaire scores related to pain or other symptoms are not lowered in fibromyalgia patients until they become residual or nonexistent. Current neurofeedback techniques cannot eliminate chronic pain completely. In particular, women with this pathology present certain social and gender biases, which sometimes make them express pain with a greater magnitude or in very different ways to eliminate this gap, as affirmed by various studies (Werner, Isaksen \& Malterud 2004; Saunders, Bartlam, Artus \& Konstantinou, 2018). Finally, another possible bias is that the majority of people diagnosed with fibromyalgia syndrome are mainly women, which could be due to the criteria used for its diagnosis according to several authors (Wolfe, Brahler, Hinz \& Hauser, 2013). Therefore, it is necessary to address the pathology from a multidisciplinary point of view (Del RíoGonzález, García-Palacios \& Botella-Arbona, 2014).

Despite the limitations that we can find in the sample size, in our study, this variable was above average for this type of research and therapy, as can be observed according to the review on neurofeedback for the management of chronic pain by Roy, de la Vega, Jensen and Miró (2020). The study does not present patient followup, which makes it impossible to compare long-term results, since this would be highly complex and expensive. Similarly, to obtain long-term results, a minimum of maintenance sessions would be required for a long-term effect to be generated. All of these findings support the thesis that neurofeedback training with an SMR 
protocol can produce relevant changes in clinical symptoms, such as pain and modify brain activity. However, there are insufficient studies on the underlying neurophysiological processes involved during brain autoregulation training, so it would be convenient to continue with this line of work.

Based on all of the previously presented data, we can conclude that the sensorimotor rhythm protocol (SMR) favors the activation of the inhibitory mechanisms of the central nervous system. In patients with fibromyalgia, this event has a direct impact on improving the subjective perception of chronic pain, the quality of sleep and the quality of life associated with health.

\section{References}

Alonso, J., Regidor, E., Barrio, G., Prieto, L., Rodríguez. C., \& de la Fuente, L. (1998). Valores poblacionales de referencia de la versión española del Cuestionario de salud SF-36 [Population reference values of the Spanish version of the SF-36 Health Survey]. Medicina Clínica, 111, 410-416. doi: 10.1157/13074369

Aparicio, V. A., Ortega, F. B., Carbonell-Baeza, A., Cuevas A. M., Delgado-Fernández, M., \& Ruiz, J. (2013). Ansiedad, depresión y su relación con el dolor y la gravedad de la fibromialgia [Anxiety, depression and its relationship to pain and the severity of fibromyalgia]. Behavioral Psychology/Psicología Conductual, 21(2), 381-392.

Bernardy, K., Füber, N., Köllner, V., \& Häuser, W. (2010). Efficacy of cognitive-behavioral therapies in fibromyalgia syndrome: A systematic review and metaanalysis of randomized controlled Trials. The Journal of Rheumatology, 37(10), 1991-2005. doi: 10.3899/jrheum. 100104

Bidari, A., Moazen-Zadeh, E., Ghavidelparsa, B., Rahmani, S., Hosseini, S., \& Hassankhani, A. (2019). Comparing duloxetine and pregabalin for treatment of pain and depression in women with fibromyalgia: An open label randomized clinical trial. Daru, 27, 149-58. doi: 10.1007/s40199-019-00257-4

Bidonde, J., Busch, A. J., Schachter, C. L., Overend, T. J., Kim, S. Y., Góes, S. M., Boden, C., \& Foulds, H. J. (2017) Aerobic exercise training for adults with fibromyalgia. The Cochrane Database of Systematic Reviews, 21, 6. doi: 10.1002/14651858.CD012700

Buysse, D. J., Reynolds, C. F., Monk, T. H., Berman, S. R., \& Kupfer, D. J. (1989). The Pittsburgh Sleep Quality Index: A new instrument for psychiatric practice and research. Psychiatry Research, 28(2), 193-213. doi: 10.1016/0165-1781(89)90047-4

Cabo-Meseguer, A., Cerdá-Olmedo, G., \& Trillo-Mata, J. L. (2017). Fibromialgia: Prevalencia, perfiles epidemiológicos y costes económicos [Fibromyalgia: Prevalence, epidemiological profiles and economic costs]. Medicina Clínica, 179(10), 441-448. doi: 10.1016/j.medcli.2017.06.008

Caro, X., \& Winter, E. (2011). EEG biofeedback treatment improves certain attention and somatic symptoms in fibromyalgia: A pilot study. Applied Psychophysiology and Biofeedback, 36(3), 193-200. doi: 10.1007/s10484-011-9159-9

Costa, I. D., Gamundí, A., Miranda, J. G., França, L. G., de Santana, C. N., \& Montoya, P. (2017). Altered functional performance in patients with fibromyalgia. Frontiers in Human Neuroscience, 11, 14. doi: 10.3389/fnhum.2017.00014

Del Río González, E., García-Palacios, A., \& Botella-Arbona, C. (2014). Calidad de vida en fibromialgia: Influencia de factores físicos y psicológicos [Quality of life in fibromyalgia: Influence of physical and psychological factors]. Behavioral Psychology/Psicología Conductual, 22(1), 19-35. 
Egner, T., \& Gruzelier, J. (2001). Learned self-regulation of EEG frequency components affects attention and event-related brain potentials in humans. Neuroreport, 12(18), 41554159. doi: 10.1097/00001756-200112210-00058

Ferrer-Peña, R., Gil-Martínez, A., Pardo-Montero, J., Jiménez-Penick, V., Gallego-lzquierdo, T., La Touche, R., (2016). Adaptación y validación de la Escala de gradación del dolor crónico al español [Adaptation and validation of the Chronic Pain Grading Scale to Spanish]. Reumatología Clínica, 12(3), 130-138. doi: 10.1016/j.reuma.2015.07.004

Gwilym, S. E., Oag, H. C., Tracey, I., \& Carr, A. J. (2011). Evidence that central sensitisation is present in patients with shoulder impingement syndrome and influences the outcome after surgery. The Journal of Bone and Joint Surgery British, 93, 498-502. doi: 10.1302/0301-620X.93B4.25054

Howe, R., \& Sterman, M. (1972). Cortical-subcortical EEG correlates of suppressed motor behavior during sleep and waking in the cat. Electroencephalography and Clinical Neurophysiology, 32, 681-695. doi: 10.1016/0013-4694(72)90104-6

Jensen, M., Hakimian, S., Sherlin, L., \& Fregni, F. (2008). New insights into neuromodulatory approaches for the treatment of pain. The Journal of Pain, 9(3), 193-199. doi: 10.1016/j.jpain.2007.11.003

Kayıran, S., Dursun, E., Dursun, N., Ermutlu, N., \& Karamürsel, S. (2010). Neurofeedback intervention in fibromyalgia syndrome: A randomized, controlled, rater blind clinical trial. Applied Psychophysiology and Biofeedback, 35(4), 293-302. doi: 10.1007/s10484010-9135-9

Kayıran, S., Dursun, E., Ermutlu, N., Dursun, N., \& Karamürsel, S. (2007). Neurofeedback in fibromyalgia syndrome. A $\breve{g r}$, 19(3), 47-53.

Kober, S., Witte, M., Stangl, M., Väljamäe, A., Neuper, C., \& Wood, G. (2015). Shutting down sensorimotor interference unblocks the networks for stimulus processing: An SMR neurofeedback training study. Clinical Neurophysiology, 126(1), 82-95. doi: 10.1016/j.clinph.2014.03.031

Lovato, N., Miller, C. B., Gordon, C. J., Grunstein, R. R., \& Lack, L. (2019). The efficacy of biofeedback for the treatment of insomnia: A critical review. Sleep Medicine, 56, 192200. doi: 10.1016/j.sleep.2018.12.011

Miró, E. Martínez, M. P., Sánchez, A. I., Prados, G., \& Medina, A. (2011). When is pain related to emotional distress and daily functioning in fibromyalgia syndrome? The mediating roles of self-efficacy and sleep quality. British Journal of Health Psychology, 16, 799814. doi: 10.1111/j.2044-8287.2011.02016.x

Moldofsky, H. (2002). Management of sleep disorders in fibromyalgia. Rheumatic Diseases Clinics of North America, 28, 353-365. doi: 10.1016/s0889-857x(01)00012-6

Napadow, V., Kim, J., Clauw, D. J., \& Harris, R. E. (2012). Decreased intrinsic brain connectivity is associated with reduced clinical pain in fibromyalgia. Arthritis \& Rheumatology, 64(7), 2398-2403. doi: 10.1002/art.34412

Prados, G., \& Miró E. (2012). Fibromialgia y sueño: Una revisión [Fibromyalgia and sleep: A review]. Revista de Neurología, 54, 227-240.

Roy, R., de la Vega, R., Jensen, M. P., \& Miró, J. (2020). Neurofeedback for pain management: A systematic review. Frontiers in Neuroscience, 16(14), 671. doi: 10.3389/fnins.2020.00671

Royuela, A., \& Macías, J. A. (1997). Propiedades clinimétricas de la versión castellana del cuestionario de Pittsburgh [Clinimetric properties of the Spanish version of the Pittsburgh questionnaire]. Vigilia Sueño, 9, 81-94.

Sánchez, A. I, Nakakaneku, M. G., Miró, E., \& Martínez, M. P. (2021). Tratamiento multidisciplinar para la fibromialgia y el síndrome de fatiga crónica: Una revisión sistemática [Multidisciplinary treatment for fibromyalgia and chronic fatigue syndrome: 
A systematic review]. Behavioral Psychology/Psicología Conductual, 29(2), 455-488. doi: 10.51668/bp.8321214s

Saunders, B., Bartlam, B., Artus, M., \& Konstantinou, K. (2018). Biographical suspension and liminality of Self in accounts of severe sciatica. Social Science Medicine, 218, 28-36. doi: 10.1016/j.socscimed.2018.10.001

Seto, A., Han, X., Price, L. L., Harvey, W. F., Bannuru, R. R., \& Wang, C. (2019). The role of personality in patients with fibromyalgia. Clinical Rheumatology, 38(1), 149-157. doi: 10.1007/s10067-018-4316-7

Sterman, M. (2000). Basic concepts and clinical findings in the treatment of seizure disorders with EEG operant conditioning. Clinical Electroencephalography, 31(1), 45-55. doi: 10.1177/155005940003100111

Terrasa, J. L., Barros-Loscertales, A., Montoya, P., \& Muñoz, M. A. (2020). Self-regulation of SMR power led to an enhancement of functional connectivity of somatomotor cortices in fibromyalgia patients. Frontiers in Neuroscience, 14, 236. doi: 10.3389/fnins.2020.00236

Thorpe, J., Shum, B., Moore, R. A., Wiffen, P. J., \& Gilron, I. (2018). Combination pharmacotherapy for the treatment of fibromyalgia in adults. Cochrane Database System, 2(2), CD010585. doi: 10.1002/14651858.CD010585.pub2

Thought Technology (2021). Neurofeedback. https://thoughttechnology.com/neurofeedback/

Vilagut, G., Ferrer, M., Rajmil, L., Rebollo, P., Permanyer-Miralda, G., Quintana, J. M., Santed, R., Valderas, J. M., Ribera, A., Domingo-Salvany, A., \& Alonso, J. (2005). El Cuestionario de salud SF-36 español: Una década de experiencia y nuevos desarrollos [The Spanish SF-36 Health Questionnaire: A decade of experience and new developments]. Gaceta Sanitaria, 19(2), 135-150. doi: 10.1157/13074369

Von Korff, M., Ormel, J., Keefe, F. J., \& Dworkin, S. F. (1992). Grading the s verity of chronic pain. Pain, 50, 133-49. doi: 10.1016/0304-3959(92)90154-4

Ware, J. E. Jr., \& Sherbourne, C. D. (1992). The MOS 36-item Short-Form Health Survey (SF36) (I). Conceptual framework and item selection. Medical Care, 30, 473-83.

Werner, A. Isaksen, L. W., \& Malterud, K. (2004). "I am not the kind of woman who complains of everything": illness stories on self and shame in women with chronic pain. Social Science Medicine, 59(5), 1035e1045. doi: 10.1016/j.socscimed.2003.12.001

Wolfe, F., Brahler, E., Hinz, A., \& Hauser, W. (2013). Fibromyalgia prevalence, somatic symptom reporting, and the dimensionality of polysymptomatic distress: Results from a survey of the general population. Arthritis Care \& Research, 65(5), 777-85. doi: 10.1002/acr.21931

Wolfe, F., Clauw, D. J., Fitzcharles, M. A., Goldenberg, D. L., Häuser, W., Katz, R. L., Mease, P. J., Russell, A. S., Russell, I. J., \& Walitt, B. (2016). Revisions to the 2010/2011 fibromyalgia diagnostic criteria. Seminars in Arthritis and Rheumatism, 46(3), 319-319. doi: 10.1016/j.semarthrit.2016.08.012

Yunus, M. (2012). The prevalence of fibromyalgia in other chronic pain conditions. Pain Research and Treatment, 2012, 1-8. doi: 10.1155/2012/584573

RECEIVED: September 7, 2020

ACCEPTED: April 3, 2021 